\title{
Maturation and integration of adult born hippocampal neurons: signal convergence onto small Rho GTPases
}

\author{
Krishna C. Vadodaria ${ }^{1,2+}$ and Sebastian Jessberger ${ }^{1,2 *}$ \\ ${ }^{1}$ Brain Research Institute, University of Zurich, Zurich, Switzerland \\ ${ }^{2}$ Neuroscience Center Zurich, University of Zurich and ETH Zurich, Zurich, Switzerland
}

\author{
Edited by: \\ Akira Yoshii, McGovern Institute for \\ Brain Research at Massachusetts \\ Institute of Technology, USA \\ Reviewed by: \\ Linda Van Aelst, Cold Spring Harbor \\ Laboratory, USA \\ Ayumu Tashiro, Nanyang \\ Technological University, Singapore \\ *Correspondence: \\ Sebastian Jessberger, Brain \\ Research Institute, University of \\ Zurich, Winterthurerstrasse 190, \\ 8057 Zurich, Switzerland \\ e-mail: jessberger@hifo.uzh.ch \\ †Present address: \\ Krishna C. Vadodaria, Laboratory of \\ Genetics, Salk Institute for Biological \\ Studies, La Jolla, CA, USA
}

\begin{abstract}
Adult neurogenesis, restricted to specific regions in the mammalian brain, represents one of the most interesting forms of plasticity in the mature nervous system. Adult-born hippocampal neurons play important roles in certain forms of learning and memory, and altered hippocampal neurogenesis has been associated with a number of neuropsychiatric diseases such as major depression and epilepsy. Newborn neurons go through distinct developmental steps, from a dividing neurogenic precursor to a synaptically integrated mature neuron. Previous studies have uncovered several molecular signaling pathways involved in distinct steps of this maturational process. In this context, the small Rho GTPases, Cdc42, Rac1, and RhoA have recently been shown to regulate the morphological and synaptic maturation of adult-born dentate granule cells in vivo. Distinct upstream regulators, including growth factors that modulate maturation and integration of newborn neurons have been shown to also recruit the small Rho GTPases. Here we review recent findings and highlight the possibility that small Rho GTPases may act as central assimilators, downstream of critical input onto adult-born hippocampal neurons contributing to their maturation and integration into the existing dentate gyrus (DG) circuitry.
\end{abstract}

Keywords: neurogenesis, Rac1, Cdc42, RhoA, synaptic integration, spine growth, dendrite, in vivo

\section{INTRODUCTION}

Throughout lifespan new neurons are continuously born in the mammalian hippocampus. It is now widely accepted that the process of adult neurogenesis is not merely a remnant of embryonic development, but a highly responsive and regulated process that appears to be critically involved in hippocampus-dependent behavior in health and disease (Sahay and Hen, 2007; Zhao et al., 2008; Danzer, 2012). The number of newborn neurons in the adult hippocampus is not static but strongly influenced by many positive and negative stimuli that influence the neurogenic process at distinct developmental stages. Positive extrinsic regulators include physical exercise, environmental enrichment, antidepressants, and learning, whereas stress and aging negatively regulate the number of newly generated neurons (Ma et al., 2009). These stimuli are thought to impact adult neurogenesis via a number of regulatory pathways including growth factors, neurotransmitters, developmental signaling molecules, and hormones. Inspired by previous studies in the context of embryonic neurogenesis, a number of cellular and molecular mechanisms, involved in the control of neural stem/progenitor cells (NSPC) activity and subsequent integration of newborn granule cells within the adult hippocampus, have been identified (Ming and Song, 2011). Even though it has been demonstrated that the neurogenic process in the adult hippocampus shares many properties with embryonic neurogenesis, it principally differs from embryonic neurogenesis in that, NSPCs and maturing neurons are present in an entirely different (adult) environment and must integrate into a preexisting circuit, presumably in the absence of large amounts of developmental guidance cues (Conover and Notti, 2008). Thus, understanding the molecular underpinnings of how adult-born neurons integrate into the dentate gyrus (DG) circuitry requires experiments that analyze the cellular mechanisms and signaling pathways in their endogenous adult niche.

Recently, a few reports demonstrate stage-specific roles for small Rho GTPases, Cdc42, Rac1, and RhoA in adult hippocampal neurogenesis in vivo. These studies suggest an important and potentially central role for the small Rho GTPases in the maturation and integration of newborn neurons in the adult hippocampus. In this mini-review, we discuss the main findings of recent in vivo studies and then focus on discussing how known upstream intrinsic regulators (neurotrophins, neurotransmitters, developmental signaling molecules, and intermediate signaling molecules) maybe recruiting Rho GTPases for mediating their effects on neuronal maturation in adulthood. We begin by describing the maturation process of adult born hippocampal neurons and give a broad overview of neuronal Rho GTPase signaling, followed by a discussion of evidence showing how important regulators of neuronal maturation may be modulating small Rho GTPases as downstream effectors. We conclude with hypotheses on mechanisms for signal convergence and a brief discussion of how Rho GTPases may act to assimilate multiple upstream signals to decisively influence cell cytoskeleton and neuronal cytoarchitecture. For a more detailed discussion on Rho GTPase signaling in neurons, please refer to other reviews (Auer et al., 2011; Govek et al., 2011; Chen et al., 2012). 


\section{MATURATION OF ADULT-BORN HIPPOCAMPAL NEURONS}

Adult hippocampal NSPCs go through distinct stages of maturation on their way to becoming fully mature newborn granule cells (Figure 1). It is currently assumed that radial glia-like NSPCs (type 1 cells) give rise to non-radial glia-like transit amplifying precursors (type 2 cells) that divide and generate immature neurons. These immature neurons grow an apical dendrite towards the molecular layer and send axonal processes to their target area, the CA3, several days after they are born (Zhao et al., 2006) (Figure 1A). During this period of maturation, newborn cells display distinct electrical properties, including gammaaminobutyric acid (GABA)-induced depolarization, contributing to their survival and functional integration into the adult hippocampal circuitry (Ge et al., 2006) (Figures 1B,C). As these neurons further mature, they start receiving excitatory synaptic input, develop dendritic spines, and display extensive dendritic arborization. Adult-generated young granule cells display hyperexcitability as compared to granule cells generated during development (Wang et al., 2000; Schmidt-Hieber et al., 2004; MarinBurgin et al., 2012). This property conceivably enables cohorts of newborn neurons to encode time (temporal context) within memory and allows the separation of patterns that are closely related, spatially or temporally (Aimone et al., 2010; Deng et al., 2013). In the rodent DG, it takes $\sim 8$ weeks for adult-born granule cells to become nearly indistinguishable from developmentallygenerated granule cells (Laplagne et al., 2006). Notably, each successive developmental stage is sensitive to a number of extrinsic and intrinsic regulators (Zhao et al., 2008; Ming and Song, 2011).

Molecular regulators of maturation and integration of adultborn neurons include neurotrophins such as brain derived neurotrophic factor (BDNF), neurotransmitters such as GABA and glutamate, and signaling molecules like Disrupted-inSchizophrenia 1 (DISC1) (Figure 1C). These regulators recruit diverse downstream pathways to finally influence distinct aspects of neuronal maturation such as migration, dendritic arborization, spine maturation and synaptic integration of newborn hippocampal neurons (Jagasia et al., 2006; Hagg, 2007). Thus, one may speculate that these molecules and their downstream effectors may partially impinge on some common signaling pathways to influence neuronal maturation. Several lines of evidence indicate that the small Rho GTPases are important and central regulators of cell cytoarchitecture in different cell types and play important roles in modulating cell migration, neurite outgrowth, survival, as well as synapse formation in neurons (Govek et al., 2005; Newey et al., 2005; Watabe-Uchida et al., 2006; Svitkina et al., 2010). Hereon, we focus on how the aforementioned regulators interact with and influence small Rho GTPase signaling to possibly modulate neuronal integration in the adult hippocampus.

\section{SMALL RHO GTPase SIGNALING}

Rho GTPases are part of the larger Ras superfamily of monomeric GTPases. These small GTPases are thought to act as binary molecular switches, transducing upstream signals to downstream effectors by alternating between the "active" GTP-bound and the "inactive" GDP-bound state (Schmitz et al., 2000) (Figure 2).

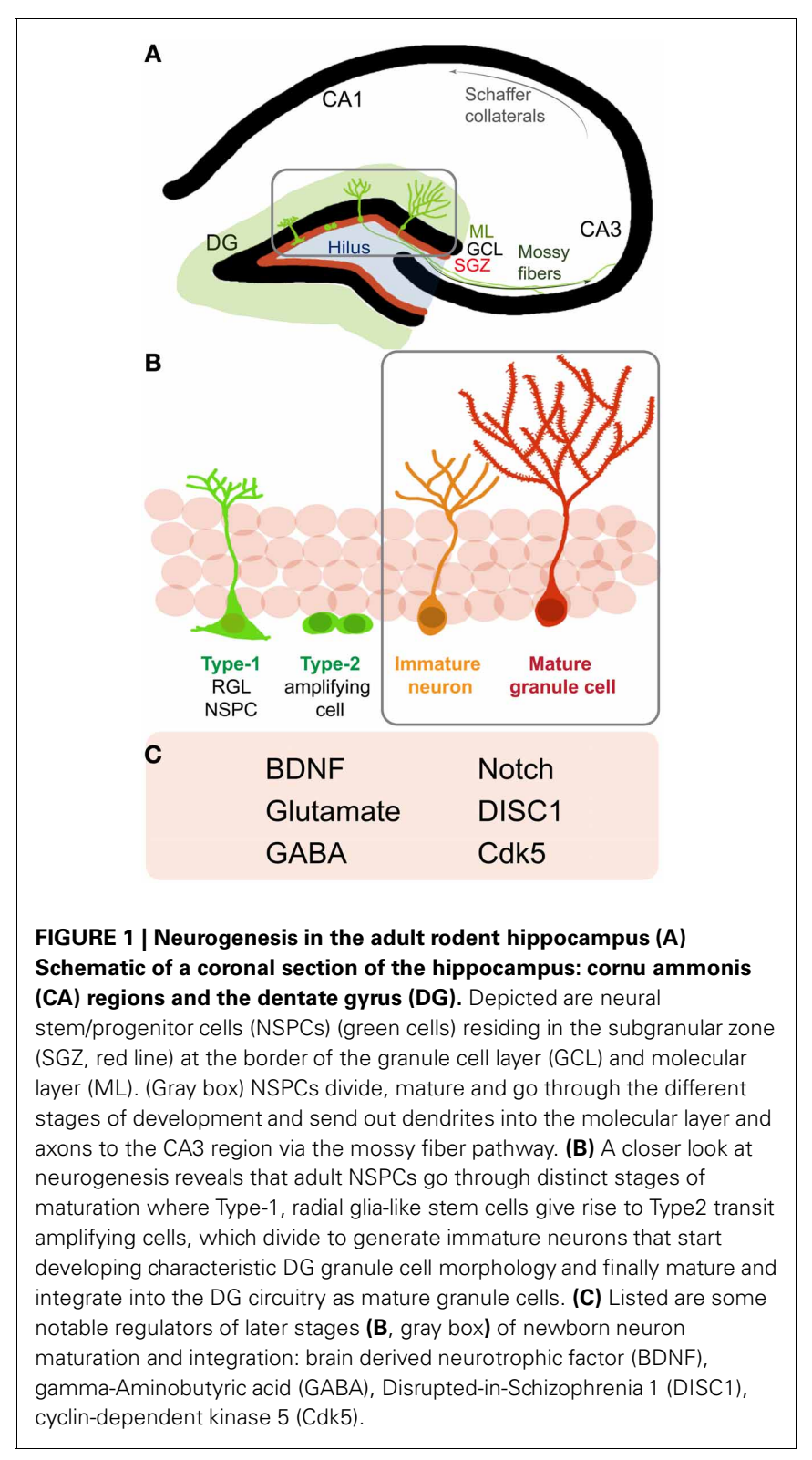

Conversion from one state to the other is tightly regulated by guanine nucleotide exchange factors (GEFs), the GTPases activating proteins (GAPs) and the guanine nucleotide dissociation inhibitors (GDIs). Small Rho GTPase activity states are regulated by the activating GEFs, which promote exchange of GDP for GTP; the inactivating GAPs (GTPases activating proteins), which enhance the intrinsic capacity of the small GTPases for hydrolyzing GTP to GDP; and the inactivating GDIs that prevent dissociation of GDP from the GTPases (as there are usually higher levels of intracellular GTP than GDP) (Jaffe and Hall, 2005). GDIs further inhibit GTPase activity by sequestering them in the cytoplasm, as opposed to the cell membrane where they functionally localize due to posttranslational lipid modifications such as prenylation and palmitoylation (Kang et al., 2008; Samuel and Hynds, 2010; Navarro-Lerida et al., 2012). GEFs, GAPs, and GDIs are specific to different Rho GTPases, and 


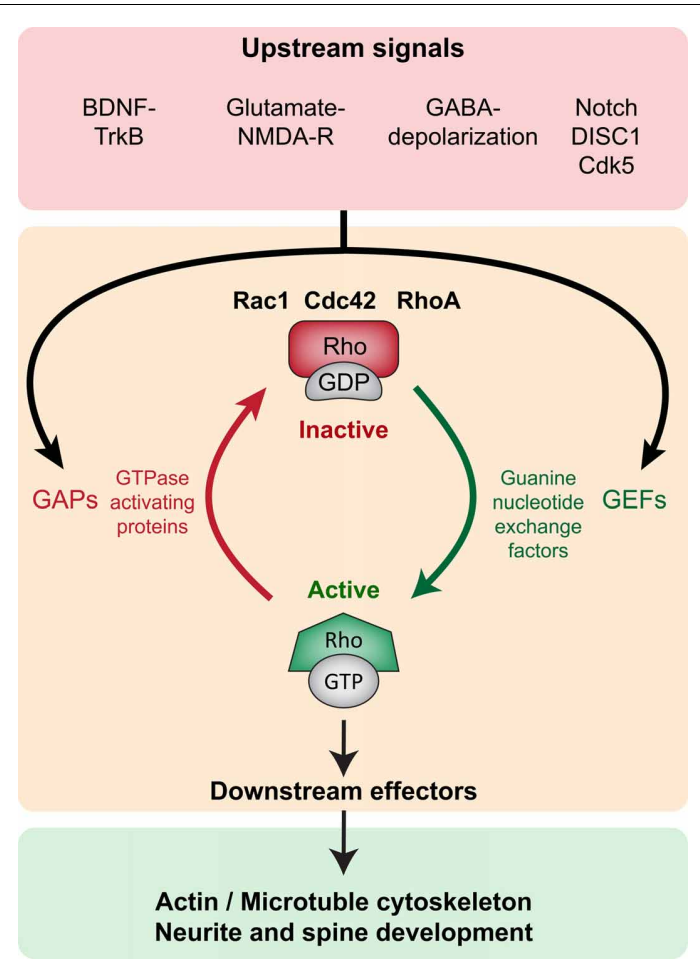

FIGURE 2 | Small Rho GTPase signaling in neurite and spine maturation (Top panel, red). Upstream signaling such as BDNF-TrkB, Glutamate-NMDAR, GABA-induced depolarization, Notch, DISC1 and Cdk5 modulate small Rho GTPase activity. (Middle panel, orange) Upstream signals modulate GEFs and GAPs thereby regulating the activity of small Rho GTPases Rac1, Cdc42, and RhoA. GEFs promote activation (GTP-bound conformation) and GAPs promote inactivation (GDP-bound conformation).

(Bottom panel, green) In the active state, small Rho GTPases bind to several downstream effectors exerting influence on local actin and microtubule networks, thereby influencing neurite and spine growth.

their expression and subcellular localization is crucial to the spatial and temporal regulation of Rho GTPase activity. Activated GTPases (GTP-bound) bind to several downstream effectors that directly modify the actin and microtubule cytoskeletal network, thereby influencing a variety of processes such as cell growth, survival, proliferation, membrane trafficking, transcriptional activation, adhesion, mechanosensation, and migration (Van Aelst and D'Souza-Schorey, 1997; Schmitz et al., 2000; Govek et al., 2005; Watabe-Uchida et al., 2006; Govek et al., 2011; Keung et al., 2011) (Figure 2).

Cdc42 (cell division cycle 42), Rac1 (ras-related C3 botulinum toxin substrate 1) and RhoA (ras homolog family member A) are the best-studied members of the small Rho GTPase family, especially in the neuronal context. Despite early indications of important roles of the Rho GTPases in neuronal maturation in vitro, there are far fewer reports examining their role in the mammalian CNS in vivo (Heasman and Ridley, 2008). This is due to the fact that straight knockouts of Cdc42 and Racl are lethal during embryogenesis, with death occurring by E9.5 (Sugihara et al., 1998; Chen et al., 2000). Newer studies, using region-specific conditional deletion of the small GTPases during development, demonstrate diverse roles for Cdc42, Rac1 and RhoA in embryonic neurogenesis and neuronal maturation (Luo et al., 1996; Cappello et al., 2006, 2012; Chen et al., 2007; De Curtis, 2008; Fuchs et al., 2009; Haditsch et al., 2009; Leone et al., 2010; Vaghi et al., 2012). Only very recently have studies begun investigating the role of the small GTPases in the adult CNS in vivo. These studies show roles for Rho GTPase signaling in SVZ/OB (Ding et al., 2010; Leong et al., 2011) and hippocampal neurogenesis (Keung et al., 2011; Christie et al., 2013; Vadodaria et al., 2013). Here, we focus on recent findings demonstrating specific roles for Rho GTPases in adult hippocampal neurogenesis.

In their study, using retrovirus mediated overexpression of constitutively active (CA, GTP-bound) and dominant negative (DN, GDP-bound) forms of RhoA in adult hippocampal neurons in vivo, Keung et al. found an inverse relationship between RhoA activity and the percentage of cells differentiating into neurons (Keung et al., 2011). However, it is possible that this effect on neuronal differentiation was due to an effect on survival of newborns and not differentiation per se, as shown recently by Christie et al. Here, the authors blocked RhoA signaling pharmacologically in vivo and observed enhanced spatial memory in the Y-maze along with an increase in newborn neuron survival, and no differences in NSPC proliferation or differentiation (Christie et al., 2013). These findings suggest that RhoA may have a "negative" role in neuronal survival and maturation. However, due to offtarget effects of DN/CA forms and pharmacological inhibitors, additional studies genetically knocking down RhoA in vivo, would help clarify its precise role in adult hippocampal neurogenesis. In our study, using both genetic deletion in vivo and $\mathrm{DN}$-form overexpression, we found stage-specific roles for Cdc42 and Rac1 in adult hippocampal neurogenesis. Cdc42 activity was found to be important for normal levels of proliferation, as well as dendritic and spine maturation. In contrast, Racl was found to be specifically important for later stages of dendritic and spine maturation (Vadodaria et al., 2013). Collectively, results from the above mentioned studies suggest that $\mathrm{Cdc} 42$ and Racl are involved in proliferation, and dendritic and spine maturation, whereas RhoA may inhibit survival and growth of newborn neurons. In addition to playing a role in adult hippocampal neurogenesis, Rac1 and its epigenetic regulation was found to regulate synaptic plasticity and spine remodeling in the adult nucleus accumbens, where it is functionally involved in addiction (Dietz et al., 2012).

Taken together, these findings hint at stage-specific roles of the Rho GTPases in adult hippocampal neurogenesis. This may be due to differential recruitment of the Rho GTPases by upstream factors during different stages of neuronal maturation. We next discuss how upstream signaling molecules may recruit Cdc42, Rac1, and RhoA, for mediating their effects on maturation and integration of newborn neurons in the adult hippocampus.

\section{REGULATORS OF NEURONAL MATURATION: TIES WITH SMALL RHO GTPase SIGNALING NEUROTROPHIN SIGNALING: BDNF}

BDNF is among the most well studied neurotrophins and has been shown to have a specific role in maturation and integration of newborn neurons in the adult hippocampus (Schmidt and Duman, 2007). Conditional mutants lacking BDNF exhibit a 
specific defect in newborn neuron maturation, but not in proliferation, cell fate specification, or survival. In particular, loss of BDNF leads to an accumulation of immature neurons with significantly shorter dendrites (Chan et al., 2008). Although BDNF binds multiple Trk receptors as well as the non-specific p75 neurotrophin receptor (NTR), it appears that the effects of BDNF deletion on neuronal morphology are likely mediated by its main receptor, $\operatorname{TrkB}$ (tropomyosin receptor kinase B). Conditional deletion of TrkB in adult NSPCs leads to a specific reduction in dendritic arborization and spine density (Bergami et al., 2008). Further, stimuli such as stress, exercise, and antidepressants that are known to regulate adult neurogenesis (and hippocampus-dependent behavior) act via regulation of BDNF signaling (Duman and Monteggia, 2006). These studies suggest an indispensible role for BDNF-TrkB signaling in the maturation and integration of newborn neurons in the adult hippocampus.

The above-mentioned in vivo studies shed light on BDNF's role in hippocampal neuron maturation, and the molecular mechanisms downstream of BDNF-TrkB signaling have been explored mainly using primary cultures in vitro. Several studies have established Rho GTPases to be downstream of TrkB activation. BDNF-TrkB signaling has been shown to specifically modulate cell morphology of hippocampal neurons in vitro, via activation of GEF Tiam1 and Rac1, and through Cdk5 dependent activation of Cdc42 (Miyamoto et al., 2006; Cheung et al., 2007). In addition to directly targeting $\mathrm{Cdc} 42$ and $\mathrm{Rac} 1, \operatorname{TrkB}$ also regulates dendritic morphogenesis by activating geranylgeranyltransferaseI, which performs lipid modifications (prenylation) of the Rho GTPases, enabling their localization at the cell membrane (Zhou et al., 2008). This may be particularly relevant as recruitment of Racl to lipid rafts in hippocampal neurons is crucial to the biological activity of neurotrophins like, nerve growth factor (Fujitani et al., 2005). These results suggest that BDNF-TrkB signaling recruits and activates the positive regulators of neurite outgrowth Cdc42 and Racl. BDNF and its protein precursor proBDNF bind to the non-specific neurotrophin receptor p75 (p75NTR), which has been shown to negatively impact cell survival and neurite outgrowth (Nykjaer et al., 2005; Blochl and Blochl, 2007). Interestingly, proBDNF-p75NTR signaling promotes growth cone collapse via downstream activation of RhoA (Sun et al., 2012). This fits in well with the notion that RhoA generally promotes growth cone collapse, whereas Cdc42 and Rac1 promote neurite growth and stabilization (Huang and Reichardt, 2003). Taken together, these studies indicate a strong link between BDNF-TrkB signaling and the regulation of small Rho GTPases for modulation of neurite growth dynamics. Given this, it is possible to hypothesize that Cdc42, Racl and RhoA may be recruited in a similar fashion downstream of BDNF for maturation of adult-born hippocampal neurons in vivo.

\section{NEUROTRANSMITTER SIGNALING: GLUTAMATE AND GABA}

Neuronal activity is central to the development of neurons, and neurotransmitters have been shown to regulate distinct stages of maturation of adult-born neurons (Vaidya et al., 2007). Glutamate and GABA primarily regulate excitation and inhibition in the adult nervous system, respectively, and have been shown to play important roles in the maturation of adult-born hippocampal neurons. Previous studies using pharmacological tools have demonstrated the importance of activity in neuronal maturation, and a key role for N-Methyl-D-aspartic acid receptor (NMDAR)-driven excitation in neuronal maturation has since long been proposed (Brewer and Cotman, 1989; McKinney et al., 1999; Richards et al., 2005; Nacher and McEwen, 2006). More recent studies have capitalized on the development of finer molecular biology tools to examine the stage-specific roles of glutamate and GABA in distinct stages of neuron maturation. Tashiro et al. showed that NMDAR-driven excitation is critical for survival and integration of newborn neurons using retrovirus-mediated single-cell knockout of NMDAR in adult-born hippocampal neurons, (Tashiro et al., 2006). Not only is glutamatergic excitation important but also GABA-induced depolarization is crucial during early stages of newborn neuron maturation. Early excitatory GABAergic input critically regulates the survival and maturation of adult-born hippocampal neurons (Ge et al., 2006, 2008). The effects of early GABAergic input on newborn neurons are likely mediated via cAMP response element-binding protein (CREB) signaling (Jagasia et al., 2009). Furthermore, recent studies have revealed which GABA receptors and subunits are involved in mediating the effects of GABA on the newborn progenitors. Adult-born hippocampal neurons of mutant mice lacking the $\alpha 4$-subunit-containing $\mathrm{GABA}_{\mathrm{A}}$ Rs and $\alpha 2$-subunit-containing $\mathrm{GABA}_{\mathrm{A}}$ Rs show defects in dendritic arborization, and cell migration in a temporally distinct manner (Duveau et al., 2011). This differs from the role of $\gamma 2$-subunit-containing $\mathrm{GABA}_{\mathrm{A}} \mathrm{Rs}$, which regulate early stages of stem cell division, as NSPCs lacking the $\gamma 2$-subunit exit quiescence and exhibit increased symmetrical divisions (Song et al., 2012). Collectively, these results indicate that glutamatergic and GABAergic input play important roles in the maturation of newborn hippocampal neurons in vivo.

The small Rho GTPases appear to be regulated by both glutamatergic and GABAergic neurotransmission via specific GEFs. Although glutamate initially and transiently activates RhoA (Jeon et al., 2002), longer activation of NMDAR leads to robust activation of the Rac1 GEF, Tiam1, specifically in dendrites and spines of hippocampal neurons in vitro (Tolias et al., 2005). This suggests that glutamate may transiently upregulate RhoA activity via AMPA receptors initially, whereas, longer or coincident excitation via NMDARs likely recruits Rac1 for mediating its positive influence on neurite growth and spine motility (Tashiro and Yuste, 2008). This is significant given that localized activation of the Rho GTPases has been directly linked to plasticity at single dendritic spines (Murakoshi et al., 2011; Yasuda and Murakoshi, 2011). GABAergic signaling has also been linked to the Rho GTPases Cdc42 and Racl. Meyer et al. show that Rac1 activity is required for optimal $\mathrm{GABA}_{\mathrm{A}}$ receptor activity, as a loss of Racl activity reduced $\mathrm{GABA}_{\mathrm{A}}$ receptor activity, possibly through loss of receptor clustering and recycling (Meyer et al., 2000). The effects of GABA on neural progenitors appear to be highly specific to the subunit composition of GABA receptors, and it remains to be elucidated how distinct subunit-containing receptors recruit different Rho GTPases. Interestingly, not only does GABAergic signaling recruit the Rho GTPases, it appears that Rho GTPases can also regulate components of the GABAergic postsynapse. The Cdc42-specific GEF Collybistin has been shown 
to regulate GABAergic neurotransmission as well as clustering of the main inhibitory postsynaptic scaffolding protein Gephyrin (Tyagarajan et al., 2011; Korber et al., 2012). This raises the interesting possibility that the small GTPases may also be playing a role in feedback or homeostatic mechanisms, influencing plasticity at the inhibitory postsynapse (Papadopoulos and Soykan, 2011). Collectively these results suggest that glutamatergic and GABAergic neurotransmission may be influencing newborn neuron maturation and spine development, at least in part by modulating small Rho GTPase activity.

\section{DEVELOPMENTAL SIGNALING MOLECULES: NOTCH}

Notch signaling involves the activation of transmembrane heterodimer receptors, activated by Delta-like or Jagged, which are membrane-bound ligands present on neighboring cells. Ligand binding results in cleavage of the transmembrane domain of the notch receptor, releasing the notch intracellular domain (NICD), which translocates to the nucleus where it interacts with RBP-J to activate transcription of several genes. Notch signaling has been extensively explored during development and shown to be critical for neuronal cell-fate choice and neurite development during embryonic neurogenesis (Sestan et al., 1999; Redmond et al., 2000). Recently, studies have found Notch to also play important roles at different stages during adult neurogenesis (Ehm et al., 2010; Ables et al., 2011). In their study, using a conditional knockout of Notch 1 in adult neural stem/progenitor cells, Breunig et al. show that in addition to playing a role in proliferation and differentiation, Notch 1 is also important for dendritic arborization of newborn neurons (Breunig et al., 2007). Recent evidence indicates that non-canonical Notch signaling recruits the Trio-Racl (GEF-GTPase) complex for axon guidance in drosophila motor neurons, indicating that non-canonical Notch signaling may be specifically involved in neurite development via modulation of small Rho GTPase activity as compared to canonical Notch signaling (Song and Giniger, 2011). Although it has been hypothesized that Rho GTPase signaling likely mediates the effects of canonical Notch signaling on neurite growth, this remains to be confirmed (Redmond and Ghosh, 2001).

\section{INTERMEDIATE SIGNAL REGULATORS}

Recently, other intermediate signaling molecules such as Disrupted in schizophrenia 1 (DISC1) and Cyclin-dependent kinase 5 (Cdk5) were found to regulate the maturation of adult born neurons (Wu et al., 2013). In a hallmark study, Duan et al. found that cell-autonomous downregulation of DISC1 accelerated morphological maturation and aberrant integration of newborn neurons in the adult hippocampus. Their study revealed DISC1 to be a key regulator in maintaining the pace of maturation and integration of adult-born neurons (Duan et al., 2007). Following this study, several other groups found DISC1 to also play important roles in other aspects of neuronal maturation, such as axonal targeting, cell-cell/cell-matrix adhesion, and neurite outgrowth (Hattori et al., 2010; Kvajo et al., 2011). Interestingly, DISC1 has also recently been shown to be downstream of regulators previously shown to regulate neuronal maturation, for example, NMDARs (Namba et al., 2011; Wu et al., 2013). Despite the relatively recent discovery of DISC1's role in newborn neuron maturation, some studies have reported Rac1 to be specifically downstream of DISC1 in regulating neuronal maturation. A study in primary neurons in vitro found that DISC1 anchors the Rac1-GEF Kalirin-7 to the postsynaptic density, and regulates local Racl activity and spine morphology downstream of NMDAR activation (Hayashi-Takagi et al., 2010). Another study, using a heterologous DISC1 transgenic system in C. elegans motor neurons, found DISC1-mutant neurons to have abnormal axonal morphology, phenocopying Rac1-mutant defects. Further, it was found that DISC1 directly interacts with the Racl GEF, Trio, promoting Racl recruitment, suggesting that the observed axonal defects are likely due to impaired downstream Racl signaling (Chen et al., 2011). Additional studies are required to precisely characterize the role of small GTPases downstream of DISC1 in regulating neuronal maturation of adult-born hippocampal neurons.

Cdk5 has been shown to phosphorylate a number of neuronal proteins specifically involved in neuronal migration and synaptic plasticity (Jessberger et al., 2009; Lopes and Agostinho, 2011). A number of GEFs and small Rho GTPase effectors such as Ephexin1 ( $\mathrm{Fu}$ et al., 2007), Ras Guanine nucleotide release factors 1 and 2 (Kesavapany et al., 2004, 2006), and Wave1 (Kim et al., 2006; Cheung and Ip, 2007) have been shown to be phosphorylated by Cdk5. In the context of adult hippocampal neurogenesis, Cdk5 appears to regulate adult neurogenesis, both in a cell-autonomous and non-cell-autonomous way. Retrovirus mediated overexpression of dominant negative Cdk5 leads to aberrant dendritic targeting and impaired spine maturation in a significant fraction of targeted adult-born hippocampal neurons, suggesting an intrinsic role for Cdk5 in neuronal maturation (Jessberger et al., 2008; Tobias et al., 2009). On the other hand, conditional deletion of Cdk5 in the NSPC population affected survival of immature neurons, an effect phenocopied in mice lacking the activating cofactor p35 (Lagace et al., 2008). This is particularly interesting because the p35/Cdk5 complex has been shown to be present at the growth cone, where it directly associates with Racl, hyperphosphorylating its effector Pak1 kinase, resulting in an attenuation of Racl signaling (Nikolic et al., 1998). Interestingly, a recent study has placed Cdk 5 downstream of BDNF in dendritic spines, in vitro, by showing that TrkB phosphorylation at specific serine residues ( $\mathrm{S} 478$ ) is Cdk5 dependent. This phosphorylation of TrkB at S478 regulates its interaction with Rac1-GEF TIAM1, and downstream Racl activation (Lai et al., 2012). These results suggest that small Rho GTPases are downstream of Cdk5 signaling, and that Cdk5 may have distinct roles in adult neurogenesis via the differential recruitment of specific GEFs and GAPs. Taken together, data from these studies indicate strong links between DISC1, Cdk5, and Racl signaling in modulating neurite outgrowth dynamics in vitro, allowing us to speculate about such interactions in vivo.

\section{MECHANISMS FOR SIGNAL CONVERGENCE IN ADULT-BORN HIPPOCAMPAL NEURONS}

Research over the last two decades has given us a large amount of information regarding the molecular mediators downstream of diverse cellular inputs. We now understand how an upstream 
activator may have diverse effects on cellular behavior by recruiting multiple signaling cascades. In the context of maturation and integration of adult-born neurons, cellular behaviors such as neurite and spine growth are readouts, used as indicators of the state in which the cell is, as well as key endpoints with functional relevance. Neurite outgrowth and spine morphogenesis, at their core, are regulated by subcellular events leading to the stabilization, extension, or collapse of local cytoskeletal elements. Therefore, despite simultaneous activation of multiple signaling pathways, decisions of actual neurite growth or retraction are consequences of single subcellular events via modulation of local microtubules or actin filaments. In vivo, newborn neurons receive diverse input, each of which may simultaneously activate different pathways that can "instruct" neurite growth or neurite retraction. However, given the limited number of potential outcomes in neurite dynamics (i.e., either growth, stability, or collapse), we hypothesize that there must be mechanisms that allow for the convergence of multiple pathways leading to a single decision resulting in either neurite extension, stabilization, or collapse at a given time.

Studies exploring different signaling pathways have revealed how the bifurcation or divergence of signals via diverse signaling cascades enables different cellular responses to the same input, but it remains unclear how convergence of pathways might be taking place. One possible mechanism for signal convergence could be that following the peak of signal divergence some signaling cascades "funnel-in" by having fewer and fewer downstream targets until they directly influence cell neurite growth. For example, pathways such as mitogen-activated protein (MAP) kinase and CREB are common targets of many upstream regulators and have been hypothesized to act as signal integrators for certain cellular processes (Wagner and Nebreda, 2009; Merz et al., 2011). Given that an upstream input can recruit different signaling entities and the fact that there are limited context-specific cellular responses that can occur, there exists a dichotomy between high signal divergence initially followed by signal convergence, resulting in a single cellular event. Spatial and temporal segregation of signaling cascades obviously enables such divergence to take place. On the same note, spatial and temporal segregation can also facilitate convergence by promoting interaction of pathways within designated spatial and temporal contexts, for example at the post-synaptic density (Sheng and Kim, 2011). Similar concepts of signal convergence for pathways involving insulin, DAG/PKC, TGF-beta/BMP, G-proteins Gz/Gi have been previously discussed (Ho and Wong, 2001; Yang and Kazanietz, 2003; Taniguchi et al., 2006).

Central decision-making moieties are another mechanism through which signal convergence may occur. Among others,

\section{REFERENCES}

Ables, J. L., Breunig, J. J., Eisch, A. J., and Rakic, P. (2011). Not(ch) just development: notch signalling in the adult brain. Nat. Rev. Neurosci. 12, 269-283. doi: 10.1038/nrn3024

Aimone, J. B., Deng, W., and Gage, F. H. (2010). Adult neurogenesis: integrating theories and separating functions. Trends Cogn. Sci. 14, 325-337. doi: 10.1016/j.tics.2010.04.003

Auer, M., Hausott, B., and Klimaschewski, L. (2011). Rho GTPases as regulators of morphological neuroplasticity. Ann. Anat. 193, 259-266. doi: 10.1016/j.aanat.2011.02.015

small Rho GTPases, due to their switch-like nature may serve to function as decision-making entities, for modulating neurite growth or collapse. As they are known to be downstream of a variety of activators, we further speculate that multiple upstream signals impinge upon the small Rho GTPases leading to decisive events shaping neurite and spine architecture. A way by which Rho GTPases may be assimilating upstream signal is by setting an activation (GTP-bound GTPase) threshold for upstream signaling. Further, regulating the concentration and regional availability of Rho GTPases may additionally enable localized assimilation of upstream signal. Sufficient input regulating the Rho GTPases' activity may come from multiple upstream pathways, where the Rho GTPases may be facilitating detection of coincident upstream input. Whether or not this is actually the case in maturation of newborn neurons remains to be resolved.

Clearly, the small Rho GTPases Cdc42, Rac1, and RhoA have functional ties with intrinsic regulators of neuronal maturation. Given their switch-like nature and a large repertoire of GEFs and GAPs regulating their state, it is likely that they are among the "decision-making" moieties via which diverse upstream regulators maybe decisively influencing neuronal architecture in the adult brain. So far, a majority of studies exploring the role of signaling molecules on neuronal function have utilized knockdown or overexpression models, in vitro and in vivo. While these approaches have generated valuable information about the basic roles of the Rho GTPases, their dispensability (knockout or dominant-negative constructs), and/or maximum functional capacity (overexpression or constitutively active constructs), we may have missed the nuanced roles of these signaling pathways of these signaling pathways under physiological conditions in neurons. Understanding whether such convergence indeed plays a role in signal transduction leading to decisive cellular events would minimally require experiments investigating activation of endogenous small GTPases, in real time, in vivo. This would provide us with significant insights into the physiological roles of these molecules and the decisive influence of regulatory pathways in mediating specific cellular behavior in the context of adult hippocampal neurogenesis.

\section{ACKNOWLEDGMENTS}

We would like to thank Dr. Himanish Ghosh for helpful comments on the manuscript. We thank the NCCR Neural Plasticity and Repair, Swiss National Science Foundation (SNF), Zurich Neuroscience Center (ZNZ), and the EMBO Young Investigator Program. Krishna C. Vadodaria is currently supported by a postdoctoral fellowship of the SNF. We apologize to authors whose original papers could not be cited in this focused mini-review due to space constraints.

Bergami, M., Rimondini, R., Santi, S. Blum, R., Gotz, M., and Canossa, M. (2008). Deletion of $\operatorname{TrkB}$ in adult progenitors alters newborn neuron integration into hippocampal circuits and increases anxietylike behavior. Proc. Natl. Acad. Sci. U.S.A. 105, 15570-15575. doi: 10.1073/pnas.0803702105
Blochl, A., and Blochl, R (2007). A cell-biological model of p75NTR signaling. J. Neurochem. 102, 289-305. doi: $\quad 10.1111 / \mathrm{j} .1471-4159.2007$ 04496.x

Breunig, J. J., Silbereis, J., Vaccarino, F. M., Sestan, N., and Rakic, P. (2007). Notch regulates cell fate 
and dendrite morphology of newborn neurons in the postnatal dentate gyrus. Proc. Natl. Acad. Sci. U.S.A. 104, 20558-20563. doi: 10.1073/pnas.0710156104

Brewer, G. J., and Cotman, C. W. (1989). NMDA receptor regulation of neuronal morphology in cultured hippocampal neurons. Neurosci. Lett. 99, 268-273. doi: 10.1016/0304-3940(89)90458-8

Cappello, S., Attardo, A., Wu, X., Iwasato, T., Itohara, S., WilschBrauninger, M., et al. (2006). The Rho-GTPase cdc42 regulates neural progenitor fate at the apical surface. Nat. Neurosci. 9, 1099-1107. doi: 10.1038/nn1744

Cappello, S., Bohringer, C. R., Bergami, M., Conzelmann, K. K., Ghanem, A., Tomassy, G. S., et al. (2012). A radial glia-specific role of RhoA in double cortex formation. Neuron 73, 911-924. doi: 10.1016/j.neuron.2011.12.030

Chan, J. P., Cordeira, J., Calderon, G. A., Iyer, L. K., and Rios, M. (2008). Depletion of central BDNF in mice impedes terminal differentiation of new granule neurons in the adult hippocampus. Mol. Cell. Neurosci. 39, 372-383. doi: 10.1016/j.mcn.2008.07.017

Chen, C., Wirth, A., and Ponimaskin, E. (2012). Cdc42: an important regulator of neuronal morphology. Int. J. Biochem. Cell Biol. 44, 447-451. doi: 10.1016/j.biocel.2011.11.022

Chen, F., Ma, L., Parrini, M. C., Mao, X., Lopez, M., Wu, C., et al. (2000). Cdc42 is required for PIP(2)-induced actin polymerization and early development but not for cell viability. Curr. Biol. 10, 758-765. doi: 10.1016/S0960-9822(00)00571-6

Chen, L., Liao, G., Waclaw, R. R., Burns, K. A., Linquist, D., Campbell, K., et al. (2007). Racl controls the formation of midline commissures and the competency of tangential migration in ventral telencephalic neurons. J. Neurosci. 27, 3884-3893. doi: 10.1523/JNEUROSCI.3509-06.2007

Chen, S. Y., Huang, P. H., and Cheng, H. J. (2011). Disruptedin-Schizophrenia 1-mediated axon guidance involves TRIORAC-PAK small GTPase pathway signaling. Proc. Natl. Acad. Sci. U.S.A. 108， 5861-5866. doi: 10.1073/pnas. 1018128108

Cheung, Z. H., Chin, W. H., Chen, Y., Ng, Y. P., and Ip, N. Y. (2007). Cdk5 is involved in BDNF-stimulated dendritic growth in hippocampal neurons. PLoS Biol. 5:e63. doi: 10.1371/journal.pbio.0050063
Cheung, Z. H., and Ip, N. Y. (2007). The roles of cyclin-dependent kinase 5 in dendrite and synapse development. Biotechnol. J. 2, 949-957. doi: 10.1002/biot.200700056

Christie, K. J., Turbic, A., and Turnley, A. M. (2013). Adult hippocampal neurogenesis, Rho kinase inhibition and enhancement of neuronal survival. Neuroscience 247C, 75-83. doi: 10.1016/j.neuroscience. 2013.05.019

Conover, J. C., and Notti, R. Q. (2008). The neural stem cell niche. Cell Tissue Res. 331, 211-224. doi: 10.1007/s00441-007-0503-6

Danzer, S. C. (2012). Depression, stress, epilepsy and adult neurogenesis. Exp. Neurol. 233, 22-32.

De Curtis, I. (2008). Functions of Rac GTPases during neuronal development. Dev. Neurosci. 30, 47-58. doi: 10.1159/000109851

Deng, W., Mayford, M., and Gage, F. H. (2013). Selection of distinct populations of dentate granule cells in response to inputs as a mechanism for pattern separation in mice. Elife 2:e00312. doi: 10.7554/eLife.00312

Dietz, D. M., Sun, H., Lobo, M. K., Cahill, M. E., Chadwick, B., Gao, V., et al. (2012). Racl is essential in cocaine-induced structural plasticity of nucleus accumbens neurons. Nat. Neurosci. 15, 891-896. doi: 10.1038/nn.3094

Ding, J., Li, Q. Y., Yu, J. Z., Wang, X., Sun, C. H., Lu, C. Z., et al. (2010). Fasudil, a Rho kinase inhibitor, drives mobilization of adult neural stem cells after hypoxia/reoxygenation injury in mice. Mol. Cell. Neurosci. 43, 201-208. doi: 10.1016/j.mcn.2009. 11.001

Duan, X., Chang, J. H., Ge, S., Faulkner, R. L., Kim, J. Y., Kitabatake, Y., et al. (2007). Disrupted-In-Schizophrenia regulates integration of newly generated neurons in the adult brain. Cell 130, 1146-1158. doi: 10.1016/j.cell.2007.07.010

Duman, R. S., and Monteggia, L. M. (2006). A neurotrophic model for stress-related mood disorders. Biol. Psychiatry 59, 1116-1127. doi: 10.1016/j.biopsych.2006.02.013

Duveau, V., Laustela, S., Barth, L., Gianolini, F., Vogt, K. E., Keist, R., et al. (2011). Spatiotemporal specificity of GABAA receptormediated regulation of adult hippocampal neurogenesis. Eur. J. Neurosci. 34, 362-373. doi: 10.1111/j.1460-9568.2011.07782.x

Ehm, O., Goritz, C., Covic, M. Schaffner, I., Schwarz, T. J., Karaca, E., et al. (2010).
RBPJkappa-dependent signaling is essential for long-term maintenance of neural stem cells in the adult hippocampus. J. Neurosci. 30, 13794-13807. doi: 10.1523/ JNEUROSCI.1567-10.2010

$\mathrm{Fu}, \mathrm{W}$. Y., Chen, Y., Sahin, M., Zhao, X. S., Shi, L., Bikoff, J. B., et al. (2007). Cdk5 regulates EphA4-mediated dendritic spine retraction through an ephexin1-dependent mechanism. Nat. Neurosci. 10, 67-76. doi $10.1038 / \mathrm{nn} 1811$

Fuchs, S., Herzog, D., Sumara, G., Büchmann-Møller, S., Civenni, G., Wu, X., et al. (2009). Stagespecific control of neural crest stem cell proliferation by the small rho GTPases Cdc42 and Rac1. Cell Stem Cell 4, 236-247. doi: 10.1016/j.stem.2009.01.017

Fujitani, M., Honda, A., Hata, K., Yamagishi, S., Tohyama, M., and Yamashita, T. (2005). Biological activity of neurotrophins is dependent on recruitment of Racl to lipid rafts. Biochem. Biophys. Res. Commun. 327, 150-154. doi 10.1016/j.bbrc.2004.11.151

Ge, S., Goh, E. L., Sailor, K. A. Kitabatake, Y., Ming, G. L., and Song, H. (2006). GABA regulates synaptic integration of newly generated neurons in the adult brain. Nature 439, 589-593. doi: 10.1038/nature04404

Ge, S., Sailor, K. A., Ming, G. L., and Song, H. (2008). Synaptic integration and plasticity of new neurons in the adult hippocampus. J. Physiol. 586, 3759-3765. doi: 10.1113/jphysiol.2008.155655

Govek, E. E., Hatten, M. E., and Van Aelst, L. (2011). The role of Rho GTPase proteins in CNS neuronal migration. Dev. Neurobiol. 71 528-553. doi: 10.1002/dneu.20850

Govek, E. E., Newey, S. E., and Van Aelst, L. (2005). The role of the Rho GTPases in neuronal development. Genes Dev. 19, 1-49. doi: 10.1101/gad.1256405

Haditsch, U., Leone, D. P., Farinelli, M. Chrostek-Grashoff, A., Brakebusch, C., Mansuy, I. M., et al. (2009). A central role for the small GTPase Racl in hippocampal plasticity and spatial learning and memory. Mol. Cell. Neurosci. 41, 409-419. doi 10.1016/j.mcn.2009.04.005

Hagg, T. (2007). Endogenous regulators of adult CNS neurogenesis. Curr. Pharm. Des. 13, 1829-1840. doi: 10.2174/138161207780858393

Hattori, T., Shimizu, S., Koyama, Y., Yamada, K., Kuwahara, R., Kumamoto, N., et al. (2010). DISC1 regulates cell-cell adhesion, cell-matrix adhesion and neurite outgrowth. Mol. Psychiatry 15, 778, 798-809. doi: 10.1038/mp.2010.60

Hayashi-Takagi, A., Takaki, M., Graziane, N., Seshadri, S., Murdoch, H., Dunlop, A. J., et al. (2010). Disrupted-in-Schizophrenia 1 (DISC1) regulates spines of the glutamate synapse via Racl. Nat. Neurosci. 13, 327-332. doi: 10.1038/nn.2487

Heasman, S. J., and Ridley, A. J. (2008). Mammalian Rho GTPases: new insights into their functions from in vivo studies. Nat. Rev Mol. Cell Biol. 9, 690-701. doi: 10.1038/nrm2476

Ho, M. K., and Wong, Y. H. (2001). $\mathrm{G}(\mathrm{z})$ signaling: emerging divergence from $G(i)$ signaling. Oncogene 20, 1615-1625. doi: 10.1038/sj.onc. 1204190

Huang, E. J., and Reichardt, L. F. (2003). Trk receptors: roles in neuronal signal transduction. Annu. Rev. Biochem. 72, 609-642. doi: $10.1146 /$ annurev.biochem.72 121801.161629

Jaffe, A. B., and Hall, A. (2005). Rho GTPases: biochemistry and biology. Annu. Rev. Cell Dev. Biol. 21, 247-269. doi: 10.1146/ annurev.cellbio.21.020604.150721

Jagasia, R., Song, H., Gage, F. H., and Lie, D. C. (2006). New regulators in adult neurogenesis and their potential role for repair. Trends Mol. Med. 12, 400-405. doi: 10.1016/j.molmed.2006.07.006

Jagasia, R., Steib, K., Englberger, E., Herold, S., Faus-Kessler, T., Saxe, M., et al. (2009). GABA-cAMP response element-binding protein signaling regulates maturation and survival of newly generated neurons in the adult hippocampus. J. Neurosci. 29, 7966-7977. doi: 10.1523/JNEUROSCI.105409.2009

Jeon, S., Kim, S., Park, J. B., Suh, P. G., Kim, Y. S., Bae, C. D., et al. (2002) RhoA and Rho kinase-dependent phosphorylation of moesin at Thr-558 in hippocampal neuronal cells by glutamate. J. Biol. Chem. 277, 16576-16584. doi: $10.1074 / j b c$ M110380200

Jessberger, S., Aigner, S., Clemenson, G. D., Toni, N., Lie, D. C., Karalay, O. et al. (2008). Cdk5 regulates accurate maturation of newborn granule cells in the adult hippocampus. PLoS Biol. 6:e272. doi: 10.1371/journal.pbio.0060272

Jessberger, S., Gage, F. H., Eisch, A. J., and Lagace, D. C. (2009). Making a neuron: cdk5 in embryonic and adult neurogenesis. Trends Neurosci. 32, 575-582. doi: 10.1016/j.tins.2009.07.002 
Kang, R., Wan, J., Arstikaitis, P., Takahashi, H., Huang, K., Bailey, A. O., et al. (2008). Neural palmitoyl-proteomics reveals dynamic synaptic palmitoylation. Nature 456, 904-909. doi: 10.1038/nature07605

Kesavapany, S., Amin, N., Zheng, Y. L., Nijhara, R., Jaffe, H., Sihag, R., et al. (2004). p35/cyclin-dependent kinase 5 phosphorylation of ras guanine nucleotide releasing factor 2 (RasGRF2) mediates Rac-dependent Extracellular Signal-regulated kinase $1 / 2$ activity, altering RasGRF2 and microtubule-associated protein $1 \mathrm{~b}$ distribution in neurons. J. Neurosci. 24, 4421-4431. doi: 10.1523/JNEUROSCI.0690-04.2004

Kesavapany, S., Pareek, T. K., Zheng, Y. L., Amin, N., Gutkind, J. S., Ma, W., et al. (2006). Neuronal nuclear organization is controlled by cyclindependent kinase 5 phosphorylation of Ras Guanine nucleotide releasing factor-1. Neurosignals 15, 157-173. doi: 10.1159/000095130

Keung, A. J., De Juan-Pardo, E. M., Schaffer, D. V., and Kumar, S. (2011). Rho GTPases mediate the mechanosensitive lineage commitment of neural stem cells. Stem Cells 29, 1886-1897. doi: 10.1002/stem.746

Kim, Y., Sung, J. Y., Ceglia, I., Lee, K. W., Ahn, J. H., Halford, J. M., et al. (2006). Phosphorylation of WAVE1 regulates actin polymerization and dendritic spine morphology. Nature 442, 814-817. doi: 10.1038/nature04976

Korber, C., Richter, A., Kaiser, M., Schlicksupp, A., Mukusch, S., Kuner, T., et al. (2012). Effects of distinct collybistin isoforms on the formation of GABAergic synapses in hippocampal neurons. Mol. Cell. Neurosci. 50, 250-259. doi: 10.1016/j.mcn.2012.05.006

Kvajo, M., McKellar, H., Drew, L. J., Lepagnol-Bestel, A. M., Xiao, L., Levy, R. J., et al. (2011). Altered axonal targeting and short-term plasticity in the hippocampus of Discl mutant mice. Proc. Natl. Acad. Sci. U.S.A. 108, E1349-E1358. doi: 10.1073/pnas.1114113108

Lagace, D. C., Benavides, D. R., Kansy, J. W., Mapelli, M., Greengard, P., Bibb, J. A., et al. (2008). Cdk5 is essential for adult hippocampal neurogenesis. Proc. Natl. Acad. Sci. U.S.A. 105, 18567-18571. doi: 10.1073/pnas.0810137105

Lai, K. O., Wong, A. S., Cheung, M. C., Xu, P., Liang, Z., Lok, K. C., et al. (2012). TrkB phosphorylation by $\mathrm{Cdk} 5$ is required for activity-dependent structural plasticity and spatial memory Nat. Neurosci. 15, 1506-1515. doi: 10.1038/nn.3237

Laplagne, D. A., Espósito, M. S., Piatti, V. C., Morgenstern, N. A., Zhao, C., Van Praag, H., et al. (2006). Functional convergence of neurons generated in the developing and adult hippocampus. PLoS Biol. 4:e409. doi: 10.1371/journal.pbio.0040409

Leone, D. P., Srinivasan, K., Brakebusch, C., and McConnell, S. K. (2010). The rho GTPase Racl is required for proliferation and survival of progenitors in the developing forebrain. Dev. Neurobiol. 70, 659-678.

Leong, S. Y., Faux, C. H., Turbic, A., Dixon, K. J., and Turnley, A. M. (2011). The Rho kinase pathway regulates mouse adult neural precursor cell migration. Stem Cells 29, 332-343. doi: 10.1002/ stem.577

Lopes, J. P., and Agostinho, P. (2011). Cdk5: multitasking between physiological and pathological conditions. Prog. Neurobiol. 94, 49-63. doi: 10.1016/j.pneurobio.2011. 03.006

Luo, L., Hensch, T. K., Ackerman, L., Barbel, S., Jan, L. Y., and Jan, Y. N. (1996). Differential effects of the Rac GTPase on Purkinje cell axons and dendritic trunks and spines. Nature 379, 837-840. doi: $10.1038 / 379837 \mathrm{a} 0$

Ma, D. K., Kim, W. R., Ming, G. L., and Song, H. (2009). Activitydependent extrinsic regulation of adult olfactory bulb and hippocampal neurogenesis. Ann. N.Y. Acad. Sci. 1170, 664-673. doi 10.1111/j.1749-6632.2009.04373.x

Marin-Burgin, A., Mongiat, L. A. Pardi, M. B., and Schinder, A. F. (2012). Unique processing during a period of high excitation/inhibition balance in adult-born neurons. Science 335, 1238-1242. doi: 10.1126/science. 1214956

McKinney, R. A., Luthi, A., Bandtlow, C. E., Gahwiler, B. H., and Thompson, S. M. (1999). Selective glutamate receptor antagonists can induce or prevent axonal sprouting in rat hippocampal slice cultures. Proc. Natl. Acad. Sci. U.S.A. 96, 11631-11636. doi: 10.1073/pnas.96.20.11631

Merz, K., Herold, S., and Lie, D. C. (2011). CREB in adult neurogenesis-master and partner in the development of adult-born neurons? Eur. J. Neurosci. 33, 1078-1086. doi: 10.1111/j.1460-9568.2011.07606.x
Meyer, D. K., Olenik, C., Hofmann, F., Barth, H., Leemhuis, J., Brunig, I., et al. (2000). Regulation of somatodendritic GABAA receptor channels in rat hippocampal neurons: evidence for a role of the small GTPase Rac1. J. Neurosci. 20, 6743-6751.

Ming, G. L., and Song, H. (2011). Adult neurogenesis in the mammalian brain: significant answers and significant questions. Neuron 70, 687-702. doi 10.1016/j.neuron.2011.05.001

Miyamoto, Y., Yamauchi, J., Tanoue, A., Wu, C., and Mobley, W. C. (2006). TrkB binds and tyrosine-phosphorylates Tiam1, leading to activation of Racl and induction of changes in cellular morphology. Proc. Natl. Acad. Sci. U.S.A. 103, 10444-10449. doi: 10.1073/pnas.0603914103

Murakoshi, H., Wang, H., and Yasuda R. (2011). Local, persistent activation of Rho GTPases during plasticity of single dendritic spines. Nature 472, 100-104. doi: 10.1038/nature09823

Nacher, J., and McEwen, B. S. (2006). The role of N-methyl-Dasparate receptors in neurogenesis. Hippocampus 16, 267-270. doi 10.1002/hipo.20160

Namba, T., Ming, G. L., Song, H., Waga, C., Enomoto, A., Kaibuchi, K., et al. (2011). NMDA receptor regulates migration of newly generated neurons in the adult hippocampus via DisruptedIn-Schizophrenia 1 (DISC1). J. Neurochem. 118, 34-44. doi 10.1111/j.1471-4159.2011.07282.x

Navarro-Lerida, I., Sanchez-Perales, S. Calvo, M., Rentero, C., Zheng, Y., Enrich, C., et al. (2012). A palmitoylation switch mechanism regulates Racl function and membrane organization. EMBO J. 31, 534-551. doi 10.1038/emboj.2011.446

Newey, S. E., Velamoor, V., Govek, E. E., and Van Aelst, L. (2005). Rho GTPases, dendritic structure, and mental retardation. J. Neurobiol. 64 58-74. doi: 10.1002/neu.20153

Nikolic, M., Chou, M. M., Lu, W., Mayer, B. J., and Tsai, L. H. (1998). The $\mathrm{p} 35 / \mathrm{Cdk} 5$ kinase is a neuronspecific Rac effector that inhibits Pak1 activity. Nature 395, 194-198 doi: $10.1038 / 26034$

Nykjaer, A., Willnow, T. E., and Petersen, C. M. (2005). p75NTRlive or let die. Curr. Opin. Neurobiol. 15, 49-57. doi: 10.1016/j.conb.2005.01.004

Papadopoulos, T., and Soykan, T. (2011). The role of collybistin in gephyrin clustering at inhibitory synapses: facts and open questions.
Front. Cell. Neurosci. 5:11. doi: 10.3389/fncel.2011.00011

Redmond, L., and Ghosh, A. (2001). The role of Notch and Rho GTPase signaling in the control of dendritic development. Curr. Opin. Neurobiol. 11, 111-117. doi: 10.1016/S09594388(00)00181-1

Redmond, L., Oh, S. R., Hicks, C. Weinmaster, G., and Ghosh, A. (2000). Nuclear Notch1 signaling and the regulation of dendritic development. Nat. Neurosci. 3, 30-40. doi: 10.1038/71104

Richards, D. A., Mateos, J. M., Hugel, S., De Paola, V., Caroni, P., Gahwiler, B. H., et al. (2005). Glutamate induces the rapid formation of spine head protrusions in hippocampal slice cultures. Proc. Natl. Acad. Sci. U.S.A. 102, 6166-6171. doi: 10.1073/pnas.0501881102

Sahay, A., and Hen, R. (2007). Adult hippocampal neurogenesis in depression. Nat. Neurosci. 10, 1110-1115. doi: 10.1038/nn1969

Samuel, F., and Hynds, D. L. (2010). RHO GTPase signaling for axon extension: is prenylation important? Mol. Neurobiol. 42, 133-142. doi: 10.1007/s12035-010-8144-2

Schmidt, H. D., and Duman, R. S. (2007). The role of neurotrophic factors in adult hippocampal neurogenesis, antidepressant treatments and animal models of depressive-like behavior. Behav. Pharmacol. 18, 391-418. doi: 10.1097/FBP.0b013e3282ee2aa8

Schmidt-Hieber, C., Jonas, P., and Bischofberger, J. (2004). Enhanced synaptic plasticity in newly generated granule cells of the adult hippocampus. Nature 429, 184-187. doi: 10.1038/nature02553

Schmitz, A. A., Govek, E. E., Bottner, B., and Van Aelst, L. (2000). Rho GTPases: signaling, migration, and invasion. Exp. Cell Res. 261, 1-12. doi: 10.1006/excr.2000.5049

Sestan, N., Artavanis-Tsakonas, S., and Rakic, P. (1999). Contactdependent inhibition of cortical neurite growth mediated by notch signaling. Science 286 , 741-746. doi: 10.1126/science.286. 5440.741

Sheng, M., and Kim, E. (2011) The postsynaptic organization of synapses. Cold Spring Harb. Perspect. Biol. 3. doi: 10.1101/ cshperspect.a005678

Song, J., Zhong, C., Bonaguidi, M. A., Sun, G. J., Hsu, D., Gu, Y., et al. (2012). Neuronal circuitry mechanism regulating adult quiescent neural stem-cell fate decision. Nature 489, 150-154. doi: 10.1038 /nature11306 
Song, J. K., and Giniger, E. (2011). Noncanonical Notch function in motor axon guidance is mediated by Rac GTPase and the GEF1 domain of Trio. Dev. Dyn. 240, 324-332. doi: 10.1002/dvdy.22525

Sugihara, K., Nakatsuji, N., Nakamura, K., Nakao, K., Hashimoto, R., Otani, H., et al. (1998). Racl is required for the formation of three germ layers during gastrulation. Oncogene 17, 3427-3433. doi: 10.1038/sj.onc. 1202595

Sun, Y., Lim, Y., Li, F., Liu, S., Lu, J. J., Haberberger, R., et al. (2012). ProBDNF collapses neurite outgrowth of primary neurons by activating RhoA. PLoS ONE 7:e35883. doi: 10.1371/journal. pone. 0035883

Svitkina, T., Lin, W. H., Webb, D. J., Yasuda, R., Wayman, G. A., Van Aelst, L., et al. (2010). Regulation of the postsynaptic cytoskeleton: roles in development, plasticity, and disorders. J. Neurosci. 30, 14937-14942. doi: 10.1523/JNEUROSCI.4276-10.2010

Taniguchi, C. M., Emanuelli, B., and Kahn, C. R. (2006). Critical nodes in signalling pathways: insights into insulin action. Nat. Rev. Mol. Cell Biol. 7, 85-96. doi: 10.1038/nrm1837

Tashiro, A., Sandler, V. M., Toni, N., Zhao, C., and Gage, F. H. (2006). NMDA-receptor-mediated, cell-specific integration of new neurons in adult dentate gyrus. Nature 442, 929-933. doi: 10.1038/ nature 05028

Tashiro, A., and Yuste, R. (2008). Role of Rho GTPases in the morphogenesis and motility of dendritic spines. Methods Enzymol. 439, 285-302. doi:

$10.1016 / \mathrm{S} 0076-6879(07)$

00421-1

Tobias, A., Saxena, M., and Lelievre, V. (2009). CDK5: the "pathfinder" for new born neurons in adult hippocampus? Cell Adh. Migr. 3, 319-321. doi: 10.4161/cam.3.4.9951

Tolias, K. F., Bikoff, J. B., Burette, A., Paradis, S., Harrar, D., Tavazoie, S., et al. (2005). The Racl-GEF Tiaml couples the NMDA receptor to the activity-dependent development of dendritic arbors and spines. Neuron 45, 525-538. doi: 10.1016/j.neuron.2005.01.024

Tyagarajan, S. K., Ghosh, H., Harvey, K., and Fritschy, J. M. (2011). Collybistin splice variants differentially interact with gephyrin and $\mathrm{Cdc} 42$ to regulate gephyrin clustering at GABAergic synapses. J. Cell Sci. 124, 2786-2796. doi: 10.1242/jcs.086199

Vadodaria, K. C., Brakebusch, C. Suter, U., and Jessberger, S. (2013). Stage-specific functions of the small Rho GTPases Cdc42 and Racl for adult hippocampal neurogenesis. J. Neurosci. 33, 1179-1189. doi: 10.1523/JNEUROSCI.2103-12.2013

Vaghi, V., Pennucci, R., Talpo, F., Corbetta, S., Montinaro, V., Barone, C., et al. (2012). Racl and Rac3 GTPases control synergistically the development of cortical and hippocampal GABAergic interneurons. Cereb. Cortex. doi: 10.1093/cercor/ bhs402. [Epub ahead of print].

Vaidya, V. A., Vadodaria, K. C., and Jha, S. (2007). Neurotransmitter regulation of adult neurogenesis: putative therapeutic targets. CNS Neurol. Disord. Drug Targets 6, 358-374. doi: 10.2174/18715270 7783220910
Van Aelst, L., and D'Souza-Schorey, C. (1997). Rho GTPases and signaling networks. Genes Dev. 11, 2295-2322. doi: 10.1101/gad.11.18.2295

Wagner, E. F., and Nebreda, A. R (2009). Signal integration by JNK and p38 MAPK pathways in cancer development. Nat. Rev. Cancer 9 , 537-549. doi: 10.1038/nrc2694

Wang, S., Scott, B. W., and Wojtowicz, J. M. (2000). Heterogenous properties of dentate granule neurons in the adult rat. J. Neurobiol. 42, 248-257.

Watabe-Uchida, M., Govek, E. E., and Van Aelst, L. (2006). Regulators of Rho GTPases in neuronal development. J. Neurosci. 26, 10633-10635. doi: 10.1523/JNEUROSCI.4084-06.2006

Wu, Q., Li, Y., and Xiao, B. (2013). DISC1-related signaling pathways in adult neurogenesis of the hippocampus. Gene 518, 223-230. doi: 10.1016/j.gene.2013.01.015

Yang, C., and Kazanietz, M. G. (2003). Divergence and complexities in DAG signaling: looking beyond PKC. Trends Pharmacol. Sci. 24, 602-608. doi: 10.1016/i.tips.2003.09.003

Yasuda, R., and Murakoshi, H. (2011) The mechanisms underlying the spatial spreading of signaling activity. Curr. Opin. Neurobiol. 21, 313-321. doi: 10.1016/j.conb.2011.02.008

Zhao, C., Deng, W., and Gage, F. H. (2008). Mechanisms and functional implications of adult neurogenesis. Cell 132, 645-660. doi: 10.1016/j.cell.2008.01.033

Zhao, C., Teng, E. M., Summers, R. G. Jr., Ming, G. L., and Gage, F. H. (2006). Distinct morphological stages of dentate granule neuron maturation in the adult mouse hippocampus. J. Neurosci. 26, 3-11. doi: 10.1523/JNEUROSCI.3648-05. 2006

Zhou, X. P., Wu, K. Y., Liang, B., Fu, X. Q., and Luo, Z. G. (2008). TrkB-mediated activation of geranylgeranyltransferase I promotes dendritic morphogenesis. Proc. Natl. Acad. Sci. U.S.A. 105, 17181-17186. doi: 10.1073/pnas. 0800846105

Conflict of Interest Statement: The authors declare that the research was conducted in the absence of any commercial or financial relationships that could be construed as a potential conflict of interest.

Received: 13 May 2013; accepted: 29 July 2013; published online: 20 August 2013.

Citation: Vadodaria KC and Jessberger $S$ (2013) Maturation and integration of adult born hippocampal neurons: signal convergence onto small Rho GTPases. Front. Synaptic Neurosci. 5:4. doi: 10.3389/fnsyn.2013.00004

This article was submitted to the journal Frontiers in Synaptic Neuroscience. Copyright (C) 2013 Vadodaria and Jessberger. This is an open-access article distributed under the terms of the Creative Commons Attribution License (CC BY). The use, distribution or reproduction in other forums is permitted, provided the original author(s) or licensor are credited and that the original publication in this journal is cited, in accordance with accepted academic practice. No use, distribution or reproduction is permitted which does not comply with these terms. 